\title{
ONA DIMINUTION
}

(nN CONSEQUENCE OR DISEASE)

OF THE AREA OF THE APERTURE,

BY WHICI

\section{THE LEFT AURICLE OF THE HEART}

COMMUNICATES

WITH THE VENTRICLE OF THE SAME SIDE.

BY JOHN ABERNETHY, ESQ. F. R. S.

FSBISTANT BURGEON 'TO ST. BARTHOLOMEW'S HOSPITAL, AND VICE PRERIDERT OR THIS SOCIETY.

Read Dec. 10, 1806 .

I HAVE thought this circumstance in morbid anatomy deserving the attention of the society, as I do not find it adverted to in any books treating on that subject. Such an impediment to the easy and natural transmission of blood from one cavity of the heart to the other, has occurred to me in the course of my dissections, not unfrequently, and in various degrees. The contraction has, in some instances, been so slight, that it would have escaped my observation, had I not by remarks on former cases, been prepared to expect it; whilst in other instances, the 
aperture, which ought perhaps to liave been an inch and a half in diameter, was so contracted, as to render it difficult to pass a large bougie from the auricle into the ventricle. In sucb cases, there existed a great mechanical impediment to the circulation of the blood, producing considerable enlargement of the left auricle, and a proportionate diminution in the capacity of the left ventricle.

It is about sixteen years since. I first met with a case which led me to expect such an impediment, and caused me to remark this circumstance in morbid anatomy, which till then I had never observed.I shall briefly relate the particulars of the case, and give an account of the dissection.

A young man, about nineteen years of age, was admitted into St. Bartholomew's Hospital, with a disease which had continued to increase for three years. His body and limbs were bulky. The cellular substance beneath the skin seemed tumid, and in some places anasarcous. His legs were anasarcous, even in a considerable degree. His belly was large, and seemed to contain some water, though not sufficient to occasion a perceptible undulation. His face, like the rest of his body, appeared bloated. The skin had every were a livid appearance. The veins were slightly turgid. The breathing was fiequent and laborious. He had a short cough, but with little expectoration. The pulse was uncommonly small and frequent. The turgid state of the 
veins, the livid colour, the general ædema, all seemed to indicate some impediment to the circulation of the blood; and the nature and cause of such obstruction became an object of speculation with us. In endeavouring to form an opinion on that subject, the smallness of the pulse led me to believe, that the left venticle of the heart received and projected into the arteries an unusually small quantity of blood.

Notwithstanding the respiration was laborious and frequent, it still appeared too free to admit of the supposition, that the left cavities of the heart re. ceived the blood in such small portions, in consequence of an impediment to its transinission through the lungs. I therefore conjectured, that a mechanical impediment prevented the left ventricle from receiving its due quantity from the auricle, and I could suggest no other kind of impediment, but some polypous growth or unnatural tumor. The symptoms increased in severity for the course of $a$ month, when the patient died.

\section{DISSECTION.}

The cellular substance was every where tumid with fluid, and serum was found in all the large cavities of the body. The lungs were dense, their 
vessels being turgid with blood, and their colls contained much serous fluid. The right cavities of the heart were healthy in appearance, and distended with blood. The left auricle was also filled with blood, and appeared of twice its natural magnitude. The left ventricle was small and empty. On laying open the left auricle, and removing the blood which it contained, I was. surprised to find the passage into the ventricle almost closed, apparently by the elevation of the mitral valves, nor could I pass even my little finger from one cavity into the other. On opening the ventricle to examine the communicating aperture more correctly, it was found to be of an elliptical form, and situated between the sides of the two pointed productions of the mitral valve. Its dimensions were not greater than would admit of the passage of a moderate sized bougie when compressed and adapted to the form. of the aperture. Indeed, the opening appeared to have been in some degree torn and enlarged by the attempt to pass a finger through it. The mitral valve appeared to be a little whiter, and more opake, than natural, but not in any degree so much as we frequently meet with it, when no such contraction is produced.

I shall next subnit to the society the opinions which I have formed relative to the cause and manner in which the contraction takes place; and although they may not, perhaps, appear completely satisfactory, the paper would seem to me deficient without them. The annulus venosus is a 
strong callous ring by which the auricles communicate with the ventricles. If the left cavities of the heart were injected to the utmost, the ring would be probably about one inch and a half in diameter. From this circle arises that duplicature of the membrane of the heart, called the valvula mitralis, into the pointed edges of which the chordæ tendinew are inserted. Now, if the fleshy columns which proceed from the middle of the heart are irritable, and frequently draw these tendinous chords, they will cause the two sides of the valve to approximate in a linear direction. Nay, as the base of the valve arrises from the annulus venosus, they will even produce an approximation in the sides of that aperture, and thus gradually tend to effect the kind of contraction which I have described. One cannot, however, suppose the closure of the opening to arise from such force alone, but from an alteration of structure and a new formation of parts according with the effect which this force would occasion: It might be suggested, that this was the mere effect of inflammation; but inflammation might cause thickening, or perhaps ossification of the valve, as we frequently see, without producing this contraction. When, however, inflammation takes place in the mitral valve, it will in some degree be extended to the tendinous chords which terminate in its points, and by these means to their fleshy columns, rendering them irritable, and thus producing the effect of approximating the sides of the valve in different degrees. 
. In the dissection which has been related we found evidences of iufiammation, but not considerable in degree. That the action of the heart tends to diminish the aperture between the auricle and ventricle, is I think, evident from examining the heart of an animal which has been bled to death when it is fornd in its utmost state of contraction; and then it is difficult to pass even the little finger from the auricle into the ventricle. I have, as I before said, met with various degrees of contraction in the aperture by which the two cavities of the left side of the heart communicate, in the ordinary course of dissections, and in some instances even to the extent described in the foregoing case. I knew nothing, however, of the symptoms which took place during life.

A late instance, however, has occurred in St. Bartholomew's Hospital, in which the symptoms were noted during life, and the morbid appearances after death. The dissection was made by $\mathrm{Mr}$. Barnes, the House Surgeon, whose diligence and talents are, I think, likely to render service to the profession, and whose account of the dissection $I$ here subjoin.

It will, however, be previously proper to give a brief account of the symptoms which the disease occasioned.

The patient was a woman of a thin and small form, and about thirty-eight years of age. I should 

unecority occupy the attention of the socistry was I to detail all the particulars of the case, as they wore recorded, during the patient's life. Mgny cir. cumatances related to the state of the atomagh and bowels, and to that of the pervous system. It seems only necessary to mention those which appear ppore immediately to bave arisen from the mechanical impediment to the circulation of the Llood; and thare. were (as in the former case) an extremely small and frequent pulse, purple tint of the skin, particplarly in the face; but there was no cedema, as in tho preceding case. The smallness and frequency of. the pulse increased, as the disease advanced, sp as: so runder it difficult to feel and count it

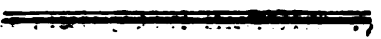

\section{DISSECTION.}

The body was opened about sixteen bqurs death. The abtouninal viscera were all in the usual state. On reflecting the sternum, the lungs appeared. of a deep purple, and loaded with blood, the pericardium tunid with fluid; on being oponed, it was found that eight or nine ounces of clear yellowisk: serum were collected. In order to examine the tharacic viscera woore minutely, they merempod. 
from their situation, by dividing the trachea and large vessels at the beginning of the neck, and again at the diaphragm. The lungs, on being cut into, were found full of a dark-coloured blood, in all parts alike; nor could the edges be made to appear white by pressing the air towards them, as is usually the case. The right cavities of the heart were empty, and perfectly sound. The left auricle appeared full, distended into a smooth surface of a solid feel, and offering considerable resistance to the finger. On slitting it open, it was found to be plugged up with coagula of a firm and fibrous texture, deposited in layers, adhering pretty strongly to the internal surface, of a moderate redness. The muscular parietes of the auricle werc increased in thickness. The annulus venosus appeared like a slit of about an inch in length and $\frac{1}{8}$ in breadth, with an irregular, hardened edge, feeling like cartilage, of a white colour, and admitting the fore-finger with difficulty. The apex of the ventricle was now cut off, to give a view of the opening below; it had here a nearly similar appearance, the mitral valves bcing much thickened, opake, and of the hardness of cartilage. It would seem that the valves were the parts most materially affected, and that the contraction was caused, as well as the shape of the opening letermined, by this circumstance.

In this case there was a much greater disease of the valve, than in the one which I first related. It is. useful to shew the different circumstances in which the contraction takes placc. I repeat that it is, as 
far as my observation euables me to determine, a frequent occurrence in various degrees. We also meet sometimes with considerable disease of the valves with but little contraction in the passage between the auricle and ventricle; and we meet with great contraction sometimes, where but little inflammation seems to have existed. These observations shew that the contraction does not depend on inflammation alone, and led me to suppose, that it was produced in the manner that $I$ have stated in a former part of the paper:

I shall next give an account to the society of an uncommon disease of the ovary. The body was examined by Mr. Hurlock, by whose means I obtained an opportunity of inspecting the diseased parts, and of relating the following particulars.

The ovary was formed into a very large, thick, and strong cyst, containing as much fluid and solid substances as filled a large pail, holding rather more than four gallons. The fluid substance resembled in colour and consistence a thick gruel made of oatmeal or arrow root. In quantity it was equal to one half of the contents of the cyst, for when poured off, the pail was half filled with solid balls of a globular form, having a smooth surface, and of the size of marbles or large bullets. There can be no doubt but that the solid balls were formed by the D 2 


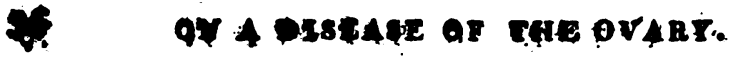

inspingation of the fuid substance, owing probably the thsorption of its thinner parts, and that they: cond their form and smaothness of sarface, in a mat mpapure, to mutual pressure and atrition; in. shaxt, that they were formed as the granules are in thatheaths of tendons. I should further mention, hent hairs grew in many places on the sides of the cyut some of which being successively detached had woorse conglabated together in a large ball, which monumed about four inches in diameter. The hairs were cemented together by the thick fluid of the ovary; but the surface of the ball had received no smooth or white cuating from it, but appeared at first sight like a ball of hair. This case is curious, rather from the rarity of its occurrence, than from its nature. A similar fluid is secreted by the sheaths of tendons and the bursa mucosæ. It becomes frm by residence in the part, and has sometimes been expressed. in this state when an opening has been made; but in general it becomes granulated by motion. It is not then wonderful that similar causes should produce similar effects, in the contents of the ovary; yet as I have not met with any record of such a case, I have been induced to communicate it to this society. 